\title{
Evolution of stars just below the critical mass for iron core formation
}

\author{
Koh Takahashi, Hideyuki Umeda and Takashi Yoshida \\ Department of Astronomy, Graduate school of Science, University of Tokyo, \\ 7-3-1 Hongo, Bunkyo-ku, Tokyo 113-0033, Japan \\ email: ktakahashi@astron.s.u-tokyo.ac.jp
}

\begin{abstract}
We calculate the evolution of stars with their initial mass of $9-11 \mathrm{M}_{\odot}$ under very fine initial mass grid of $0.01 \mathrm{M}_{\odot}$. We determine the lower critical mass for Ne ignition in an ONe core that has not undergone the thermal pulse episode. The values are $9.83 \mathrm{M}_{\odot}$ for the initial mass and $1.365 \mathrm{M}_{\odot}$ for the $\mathrm{CO}$ core mass. A star with an initial mass slightly larger than the critical, undergoes an off-center $\mathrm{Ne}+\mathrm{O}$ ignition. Since the energy production rate of $\mathrm{Ne}+\mathrm{O}$ burning and lasting electron capture reactions is sufficiently large to ignite $\mathrm{Si}$, an Fe core forms as a result of shell Si burning. For a star just below the critical mass, an ONe core continues to contract. In such a high density core, electron capture by nuclei produced through $\mathrm{C}$ burning affects the core evolution. After ${ }^{20} \mathrm{Ne}$ starts to capture electrons, the core may ignite $\mathrm{O}$ and undergo $\mathrm{O}$ detonation. The fate may be an Electron Capture Supernova.
\end{abstract}

Keywords. stars: evolution, supernovae: general, nucleosynthesis

\section{Introduction}

A star with initial mass of 9-11 $\mathrm{M}_{\odot}$ forms a $\mathrm{CO}$ core around the Chandrasekhar limit $\mathrm{M}_{\mathrm{Ch}} \simeq 1.38 \mathrm{M}_{\odot}$. While it is well known that a star with a $\mathrm{CO}$ core sufficiently larger than $\mathrm{M}_{\mathrm{Ch}}$ forms an $\mathrm{Fe}$ core after non-degenerate Si ignition and collapses (Woosley et al. 1986), in the case for a star with a CO core slightly less than the limit, the evolution after an ONe core formation is still poorly known. This is due to complicated aspects of their evolution such as thermal pulse (Poelarends et al. 2008) or convective URCA process (Ritossa et al. 2008).

However, the importance for their understanding is apparent. Until now, the only model for a supernova explosion that can be achieved in first-principle calculation is Electron Capture Supernova (ECSN) which have a progenitor of the Chandrasekhar limit mass ONe core (Nomoto 1987, Kitaura et al. 2006). We investigate detailed evolution of 9$11 \mathrm{M}_{\odot}$ stars which form degenerate ONe cores.

\section{The critical mass for Ne ignition}

Figure 1 shows evolution tracks in terms of the central temperature and density after $\mathrm{C}$ burning. We see clear differences between less massive $9.82,9.83 \mathrm{M}_{\odot}$ stars and more massive $9.9,10 \mathrm{M}_{\odot}$ stars in the tracks. For less massive stars of $9.82,9.83 \mathrm{M}_{\odot}$, as the density at the center reaches the value of $\log \rho \sim 9$, the temperature starts to decrease. This is owing to URCA cooling by ${ }^{25} \mathrm{Mg}$ and ${ }^{23} \mathrm{Na}$. On the other hand, in the tracks for more massive stars, spikes appear at $\log \rho \sim 8$ in which the core expands and contracts adiabatically due to $\mathrm{Ne}+\mathrm{O}$ or $\mathrm{Ne}+\mathrm{O}+\mathrm{Si}$ shell burning. We determine $9.83 \mathrm{M}_{\odot}$ as the lower critical initial mass for Ne ignition. The corresponding CO core mass is $1.365 \mathrm{M}_{\odot}$. Hereafter we describe some details of the evolution. 


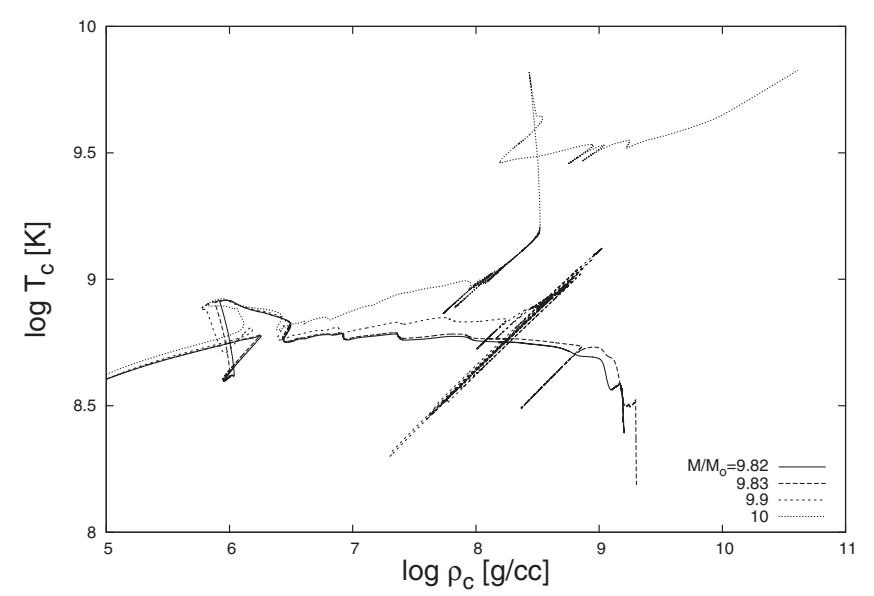

Figure 1. Evolution tracks in terms of central temperature and density.

9.82M $M_{\odot}$ model. The star with the initial mass of $9.82 \mathrm{M}_{\odot}$ forms a CO core of $1.360 \mathrm{M}_{\odot}$ and later an $\mathrm{ONe}$ core forms after semi-degenerate shell $\mathrm{C}$ burning. As the core contracts, the inverse temperature distribution comes out and the highest temperature region eventually moves outward to the edge of the core. This is the general feature which appears in a high density core supported by degenerate electrons. Finally, the core keeps contracting without Ne ignition. In the contracting ONe core, reactions of electron capture by C burning products occur in succession. After ${ }^{20} \mathrm{Ne}$ start to capture electrons, temperature at the center will increase rapidly due to a decrease in the number of electrons which support the core. As the temperature reaches the critical value to ignite $\mathrm{Ne}$ and $\mathrm{O}, \mathrm{O}$ detonation may take place at the center and the fate may be an ECSN.

$10 M_{\odot}$ model. A star with a slightly larger core undergoes shell Ne ignition and the fate will be CCSN. In the less-degenerate CO core, C ignites at the center of the $10 \mathrm{M}_{\odot} \mathrm{star}_{\text {, }}$ while for the $9.9 \mathrm{M}_{\odot}$ star, the formation of the ONe core occurs in the same way as the case of $9.82 \mathrm{M}_{\odot}$ star. Since it has a larger $\mathrm{CO}$ core, $1.49 \mathrm{M}_{\odot}$, and electrons at the center are less degenerate, off-center Ne ignition takes place. The Ne burning becomes a flash due to the high degeneracy of the core and is followed by shell Si burning, as well as by shell O burning. A strong shell burning occurs and Ne, O and Si simultaneously burn in the shell. The Fe core forms after the burning front reaches the center, and the core contracts and collapses.

Shell $\mathrm{Ne}+\mathrm{O}+\mathrm{Si}$ burning will be the general aspects for stars which have slightly larger core mass than the critical of $1.365 \mathrm{M}_{\odot}$ for the $\mathrm{CO}$ core. The star of $9.9 \mathrm{M}_{\odot}$ for its initial and $1.404 \mathrm{M}_{\odot}$ for the $\mathrm{CO}$ core also shows $\mathrm{Ne}+\mathrm{O}+\mathrm{Si}$ burning. In the case of the star of $9.83 \mathrm{M}_{\odot}$, a contracting phase exists between shell $\mathrm{Ne}+\mathrm{O}$ burning and shell Si burning. So far, the critical mass for $\mathrm{Ne}$ ignition is $9.83 \mathrm{M}_{\odot}$ for the initial mass, $1.365 \mathrm{M}_{\odot}$ for the $\mathrm{CO}$ core.

\section{References}

Kitaura, F. S., Janka, H.-Th., \& Hillebrandt, W. 2006, A\&A A, 450, 345

Nomoto, K. 1987, ApJ, 322, 206

Poelarends, A. J., Herwig, F., Langer, N., \& Heger, A. 2008, ApJ, 675, 614

Rittosa, C., Garcia-Berro, E., \& Iben, I. J. 1999, ApJ, 525, 381

Woosley, S. E. \& Weaver, T. A. 1986, ARAA, 24, 205 